\title{
SMOOTH RIGIDITY AND $C^{1}$-CONJUGACY AT $\infty$
}

\author{
F. T. FARRELL AND L. E. JONES
}

\section{INTRODUCTION}

Let $M$ and $N$ be closed (connected) non-positively curved Riemannian manifolds and $\alpha: \pi_{1}(M) \rightarrow \pi_{1}(N)$ be an isomorphism. We showed in [9] that $\alpha$ is induced by a homeomorphism at least when $\operatorname{dim} M \neq 3,4$. This result was motivated by Mostow's Strong Rigidity Theorem [23] which showed that $\alpha$ is in fact induced by an isometry if $M$ and $N$ satisfy some more geometric constraints and provided we are allowed to change the metric on $M$ by scaling it on each irreducible metric factor of its universal cover. These extra constraints are that both manifolds be locally symmetric spaces and that the universal cover of $M$ does not have a 1 or 2 dimensional metric factor. (A slightly weaker condition on the universal cover is sufficient and the condition dim $M \neq 3,4$ can be dropped.) Eberlein [5] and Gromov [2] showed Strong Rigidity still holds if Mostow's hypothesis that $N$ is locally symmetric is dropped and the hypothesis on $M$ is strengthened as follows: $M$ must have some sectional curvature equal to 0 and no finite sheeted cover of $M$ is a non-trivial metric product. The weaker conclusion of [9] is called topological rigidity and smooth rigidity would mean that $\alpha$ is induced by a diffeomorphism. We gave examples in [8] showing that smooth rigidity fails for compact non-positively (even negatively) curved manifolds. We in fact constructed a pair of compact negatively curved Riemannian manifolds $M$ and $N$ which are homeomorphic but not diffeomorphic, and additionally $M$ is a locally symmetric space and $M \times M$ is not diffeomorphic to $N \times N$. Hence neither of the two extra conditions on $M$ in the Eberlein-Gromov strong rigidity theorem can be removed

Both authors were supported in part by the National Science Foundation. 
and still even get smooth rigidity.

There is another variant of strong rigidity recently proven. In it, Mostow's condition on $M$ is strengthened to require that the sectional curvatures of $M$ are all negative but not constant. And his condition that $N$ be locally symmetric is dropped; but added is the condition that all of the sectional curvatures of $N$ lie in the interval $\left[-a^{2},-a^{2} / 4\right]$ for some number $a \neq 0$; i.e., $N$ is 1/4-pinched. This result was proven independently by Hernandez [17] and Yau-Zheng [26] when $M$ is covered by complex hyperbolic space after scaling its metric by a positive constant. When $M$ is covered (after scaling) by quaternionic hyperbolic space or the Cayley hyperbolic plane, the result follows from the work of Hernandez [17], Corlette [4] and Gromov [13]; cf. [6, p. 213]. On the other hand, we constructed examples in [10] of a homeomorphic pair of compact negatively curved Riemannian manifolds $M$ and $N$ which are not diffeomorphic. In these examples, $M$ is a locally symmetric space of nonconstant sectional curvatures and $N$ is almost $1 / 4$-pinched. In fact, given any real number $b>4$, examples are.constructed where all of the sectional curvatures of $N$ lie in the interval $[-b,-1]$ and $M$ is covered by complex hyperbolic space. Hence the 1/4-pinching condition on $N$ in the Hernandez, Yau-Zheng strong rigidity theorem cannot be removed and still even get smooth rigidity in general.

One can also ask whether topological rigidity can be improved to PL-rigidity when $M$ and $N$ are closed non-positively curved; i.e., is $\alpha$ always induced by a piecewise linear homeomorphism; i.e., by a simplicial isomorphism between piecewise smooth triangulations of $M$ and $N$ ? But again this is generally not so. Ontaneda [24] has in fact recently constructed examples of pairs of homeomorphic 6-dimensional compact negatively curved Riemannian manifolds $M$ and $N$ which are not PL-homeomorphic. Given $\epsilon>0$, he also has examples where the sectional curvatures of both $M$ and $N$ are contained in the interval $[-1-\epsilon,-1]$; in fact, $M$ is a real hyperbolic manifold.

These results motivate the following query.

Question. What extra geometric conditions will guarantee smooth (or PL) rigidity? 
Searching for an answer led us back to Mostow's original work on strong rigidity [22]. He showed there that when the actions of $\pi_{1}(M)$ and $\pi_{1}(N)$ by deck transformations on the universal covers of $M$ and $N$ are topologically conjugate and this conjugacy extends to a $C^{1}$ - conjugacy at $\infty$, then strong rigidity holds even if $M$ and $N$ are hyperbolic 2-manifolds. The assumption here is that $M$ and $N$ are both compact locally symmetric spaces of noncompact type; and at $\infty$ means on the Furstenberg maximal boundary of the corresponding symmetric spaces. When $M$ and $N$ are strictly negatively curved, the Furstenberg maximal boundary is the same as the Eberlein-O'Neill visibility sphere [7].

We now proceed to formulate our partial result Theorem 0.2 on this Question. We fix the following assumptions and notation throughout the remainder of this paper. Let $M$ and $N$ be compact (connected) non- positively curved Riemannian manifolds and let $\alpha: \pi_{1}(M) \rightarrow \pi_{1}(N)$ be an isomorphism. We also assume that $\operatorname{dim} M \geq 5$. Let $\tilde{M}$ and $\tilde{N}$ denote the total spaces of the universal covers of $M$ and $N$. Identify $\pi_{1}(M)$ and $\pi_{1}(N)$ with the groups of deck transformations of $\tilde{M}$ and $\tilde{N}$, respectively. Let $\tilde{M}(\infty)$ and $\tilde{N}(\infty)$ denote the Eberlein-O'Neill [7] visibility spheres of $\tilde{M}$ and $\tilde{N}$. Recall that a point on $\tilde{M}(\infty)$ is an asymptoty class of geodesic rays in $\tilde{M}$. Let $S \tilde{M} \rightarrow \tilde{M}$ denote the tangent sphere bundle of $\tilde{M}$. Then there is a natural map

$$
F: S \tilde{M} \rightarrow \tilde{M}(\infty)
$$

defined by $F(v)=\gamma_{v}(+\infty)$. Here $\gamma_{v}$ is the unique geodesic in $\tilde{M}$ satisfying $\dot{\gamma}_{v}(0)=v$ and $\gamma_{v}(+\infty)$ is the asymptoty class containing the geodesic ray $\left\{\gamma_{v}(t) \mid t \geq 0\right\}$. The map $F$ restricted to any fiber of $S \tilde{M} \rightarrow \tilde{M}$ is a homeomorphism onto $\tilde{M}(\infty)$. Consequently, the action of $\pi_{1}(M)$ on $\tilde{M}$ naturally induces an action on $\tilde{M}(\infty)$.

Adjoining $\tilde{M}(\infty)$ to $\tilde{M}$ gives a natural compactification $\bar{M}$ for $\tilde{M}$ where $\bar{M}$ has the cone topology in the following sense, cf. $[6$, p. 182]. Let $\omega:[0,1] \rightarrow$ $[0,+\infty]$ be any homeomorphism with $\omega(0)=0$ and $x$ be any point in $\tilde{M}$. Then 
the function

$$
\begin{aligned}
& v \mapsto \gamma_{v /|v|}(\omega(|v|)), \quad v \neq 0 \\
& 0 \mapsto x
\end{aligned}
$$

is a homeomorphism of the closed unit radius ball $B_{x} \tilde{M}$ with center 0 in $T_{x} \tilde{M}$ to $\bar{M} .\left(T_{x} \tilde{M}\right.$ denotes the tangent space to $\tilde{M}$ at $x$ and $S_{x} \tilde{M}=\partial B_{x} \tilde{M}$.) The actions of $\pi_{1}(M)$ on $\tilde{M}$ and $\tilde{M}(\infty)$ then glue together yielding an action on $\bar{M}$.

Definition 0.1. The visibility sphere $\tilde{M}(\infty)$ is naturally $C^{1}$ provided $\tilde{M}(\infty)$ has a $C^{1}$ - manifold structure such that $F: S \tilde{M} \rightarrow \tilde{M}(\infty)$ is a $C^{1}$-map and $F$ restricted to each fiber $S_{x} \tilde{M}$ of $S \tilde{M} \rightarrow \tilde{M}$ is a $C^{1}$ - diffeomorphism.

Remark 0.1 .1 . If $\tilde{M}(\infty)$ is naturally $C^{1}$, then this $C^{1}$ structure is unique and the action of $\pi_{1}(M)$ on $\tilde{M}(\infty)$ is via $C^{1}$-diffeomorphisms.

Theorem 0.2. The isomorphism $\alpha$ is induced by a smooth diffeomorphism $f: M \rightarrow N$ when the following 4 conditions all hold.

1. $\tilde{M}(\infty)$ and $\tilde{N}(\infty)$ are both naturally $C^{1}$.

2. The actions $\left(\tilde{M}(\infty), \pi_{1} M\right)$ and $\left(\tilde{N}(\infty), \pi_{1} N\right)$ are $\alpha$-equivariantly $C^{1}$ conjugate.

3. The $C^{1}$-conjugacy of condition 2 extends to an $\alpha$-equivariant $C^{0}$-semiconjugacy from $\left(\bar{M}, \pi_{1} M\right)$ to $\left(\bar{N}, \pi_{1} N\right)$.

4. The Euler characteristic $\chi(M)=0$.

Remark 0.2.1. Condition 2 of 0.2 means there is a $C^{1}$ - diffeomorphism $\phi$ : $\tilde{M}(\infty) \rightarrow \tilde{N}(\infty)$ such that $\phi(g x)=\alpha(x) \phi(x)$ for each $x \in \tilde{M}(\infty)$ and $g \in$ $\pi_{1}(M)$. Condition 3 means $\phi$ extends to a continuous map $\bar{\phi}: \bar{M} \rightarrow \bar{N}$ such that $\bar{\phi}(g x)=\alpha(g) \bar{\phi}(x)$ for all $x \in \bar{M}, g \in \pi_{1}(M)$. It is important in proving 0.2 that this semi-conjugacy $\bar{\phi}$ can be improved to be a $C^{0}$-conjugacy $\bar{\psi}$ extending $\phi$ as follows. The restriction $\left.\bar{\phi}\right|_{\tilde{M}}$ is a lift of some map $\phi_{0}: M \rightarrow N$. Because of [9], there is a homotopy $\phi_{t}: M \rightarrow N$ from $\phi_{0}$ to a homeomorphism $\phi_{1}$, $t \in[0,1]$. Let $\tilde{\phi}_{t}: \tilde{M} \rightarrow \tilde{N}$ be the lift of this homotopy such that $\tilde{\phi}_{0}=\left.\bar{\phi}\right|_{\tilde{M}}$ and note that $\tilde{\phi}_{1}$ is a homeomorphism. Define $\bar{\psi}: \bar{M} \rightarrow \bar{N}$ by $\left.\bar{\psi}\right|_{\tilde{M}}=\tilde{\phi}_{1}$ and $\left.\bar{\psi}\right|_{\tilde{M}(\infty)}=\phi ;$ cf. the paragraph preceding Corollary 0.4 . 
Recall that condition 4 of 0.2 is redundant when $\operatorname{dim} M$ is odd since odd dimensional closed manifolds have zero Euler characteristic. We proceed next to formulate an addendum to Theorem 0.2 which gives useful information in the cases where $\chi(M) \neq 0$ but conditions 1,2 and 3 are still satisfied. Recall that a homotopy $n$-sphere $\Sigma$ is a (oriented) smooth manifold which is homeomorphic to the $n$-sphere $S^{n}$. The set of all oriented diffeomorphism classes of homotopy $n$-spheres $(n \geq 5)$ is a finite abelian group under the operation \# of connected sum. This group is denoted $\Theta_{n}$ and was analyzed by Kervaire and Milnor [20].

Addendum 0.3. If conditions 1, 2 and 3 of Theorem 0.2 are satisfied, then there exists a homotopy $m$-sphere $\Sigma$ (where $m=\operatorname{dim} M$ ) and a smooth diffeomorphism $f: M \# \chi(M) \Sigma \rightarrow N$ which induces $\alpha$. In particular, there exists a PL-homeomorphism $g: M \rightarrow N$ inducing $\alpha$. (Here $M \# \chi(M) \Sigma$ denotes connected sum with $\chi(M)$-copies of $\Sigma$.)

Remark 0.3.1. The homotopy sphere $\Sigma$ of 0.3 is explicitly constructed from the $\alpha$-equivariant $C^{1}$-conjugacy $\phi: \tilde{M}(\infty) \rightarrow \tilde{N}(\infty)$ of condition 2 (cf. Remark 0.2.1) as follows. Pick points $x \in \tilde{M}, y \in \tilde{N}$ and consider the closed unit radius balls $B_{x}, B_{y}$ centered at 0 in the tangent spaces of $M, N$ at $x, y$, respectively. Then $\Sigma$ results from gluing together the boundaries of $B_{x}, B_{y}$ via the diffeomorphism which is the composition $\left(g_{y}\right)^{-1} \circ \phi \circ g_{x}$ where $g_{x}, g_{y}$ are respectively the $C^{1}$-diffeomorphisms

$$
\begin{aligned}
& \partial B_{x} \subset S \tilde{M} \rightarrow \tilde{M}(\infty), \\
& \partial B_{y} \subset S \tilde{N} \rightarrow \tilde{N}(\infty),
\end{aligned}
$$

given by condition 1 (cf. Definition 0.1). We do not know an example where this $\Sigma$ is not diffeomorphic to $S^{m}$. In fact, we conjecture that $\Sigma$ is always diffeomorphic to $S^{m}$. If so, then Theorem 0.2 remains true when condition 4 is dropped.

Throughout the rest of this introduction, we specialize to the situation where both $M$ and $N$ are strictly negatively curved. Under these stronger assumptions, Mostow showed that the actions $\left(\tilde{M}(\infty), \pi_{1} M\right)$ and $\left(\tilde{N}(\infty), \pi_{1} N\right)$ 
are always $\alpha$-equivariantly $C^{0}$-conjugate via a unique homeomorphism $\alpha_{\infty}$ : $\tilde{M}(\infty) \rightarrow \tilde{N}(\infty)$. (Pugh's Closing Lemma is used in showing uniqueness.) Furthermore, $\alpha_{\infty}$ extends to an $\alpha$-equivariant $C^{0}$-semi-conjugacy from $\left(\bar{M}, \pi_{1} M\right)$ to $\left(\bar{N}, \pi_{1} N\right)$. This semi-conjugacy is constructed as follows. Let $\psi: M \rightarrow N$ be any continuous map inducing $\alpha$ and let $\tilde{\psi}: \tilde{M} \rightarrow \tilde{N}$ be its $\alpha$-equivariant lift to the universal covering spaces. Then a semi-conjugacy $\bar{\psi}$ extending $\alpha_{\infty}$ is defined by $\left.\bar{\psi}\right|_{\bar{M}}=\tilde{\psi}$ and $\left.\bar{\psi}\right|_{\tilde{M}(\infty)}=\alpha_{\infty}$. Moreover, the map $\alpha_{\infty}$ is determined by the following property valid for each vector $v \in S \tilde{M}$. Let $\gamma_{v}$ be the geodesic such that $\dot{\gamma}_{v}(0)=v$. Then each geodesic ray in the asymptoty class of $\alpha_{\infty}\left(\gamma_{v}(+\infty)\right)$ is a finite Hausdorff distance from the set $\left\{\tilde{\psi}\left(\gamma_{v}(t)\right) \mid t \geq 0\right\}$. Note we can choose $\psi$ to be a homeomorphism because of [9]; in which case, $\bar{\psi}$ is a $C^{0}$-conjugacy. We consequently have the following strengthening of 0.2 and 0.3 under this specialization.

Corollary 0.4. Assume that both $M$ and $N$ are strictly negatively curved. Then there exists a smooth diffeomorphism

$$
f: M \# \chi(M) \Sigma \rightarrow N
$$

inducing $\alpha$ provided

1. $\tilde{M}(\infty)$ and $\tilde{N}(\infty)$ are both naturally $C^{1}$; and

2. $\alpha_{\infty}$ is a $C^{1}$-diffeomorphism.

Here $\Sigma$ is the homotopy sphere constructed from $\alpha_{\infty}$ via the procedure given in Remark 0.3.1.

Remark 0.4.1. We do not know an example where conditions 1 and 2 of 0.4 are both satisfied but $M$ and $N$ are not isometric after multiplying the metric on $M$ by a suitable constant. We hope that weaker conditions at $\infty$ than conditions 1 and 2 should imply that $M$ and $N$ are diffeomorphic.

We prove Theorem 0.2 and Addendum 0.3 in section 1. Section 2 is devoted to deducing an application. We complete this introduction by formulating this application.

We start by recalling some definitions. First, $M$ is strictly $1 / 4-$ pinched if there exists a positive real number $a$ such that all the sectional curvatures 
of $M$ lie in the open interval $(-a,-a / 4)$. Next, there is a real valued length function $l_{M}: \pi_{1}(M) \rightarrow \mathbb{R}$ which assigns to each $g \in \pi_{1}(M)$ the length $l_{M}(g)$ of the unique closed geodesic in the free homotopy class of curves determined by $g$. Then, $\alpha$ induces an isomorphism of marked length spectra provided $l_{N} \circ \alpha=l_{M}$.

It is a well known conjecture that any isomorphism of marked length spectra is induced by an isometry when the manifolds are compact and negatively curved; cf. [15], [6]. This conjecture has been verified in the important special case where one of the manifolds is a locally symmetric space. This result is due to Hamenstädt [16] when one of the manifolds is real hyperbolic; i.e., has constant negative sectional curvature. Her result was recently extended to the other negatively curved locally symmetric spaces by Besson, Courtois and Gallot [3]. Our application of Corollary 0.4 gives additional positive (albeit weak) information on this conjecture.

Theorem 0.5. Assume that both $M$ and $N$ are strictly $1 / 4$-pinched. If $\alpha$ induces an isomorphism of marked length spectra, then there exists a smooth diffeomorphism

$$
f: M \# \chi(M) \Sigma \rightarrow N
$$

inducing $\alpha$. (Here $\Sigma$ is the homotopy sphere from Corollary 0.4.) In particular, $M$ and $N$ are always $P L$-homeomorphic, and diffeomorphic when dim $M$ is odd.

Acknowledgement. We wish to thank Chris Stark and Livio Flaminio for helpful conversations concerning this paper.

\section{Proof of the MAin Result}

We assume throughout this section that conditions 1, 2 and 3 of Theorem 0.2 are satisfied. Hence there is an $\alpha$-equivariant homeomorphism $\bar{\phi}: \bar{M} \rightarrow \bar{N}$ such that

1. $\bar{\phi}(\tilde{M})=\tilde{N}$ and $\bar{\phi}(\tilde{M}(\infty))=\tilde{N}(\infty)$;

2. $\phi_{\infty}: \tilde{M}(\infty) \rightarrow \tilde{N}(\infty)$ is a $C^{1}$-diffeomorphism where $\phi_{\infty}=\left.\bar{\phi}\right|_{\tilde{M}(\infty)}$. 
Consider the $\alpha$-equivariant homeomorphism $\tilde{\phi}: \tilde{M} \rightarrow \tilde{N}$ where $\tilde{\phi}=\left.\bar{\phi}\right|_{\tilde{M}}$; it induces a homeomorphism $\phi: M \rightarrow N$. The groups $\pi_{1}(M)$ and $\pi_{1}(N)$ act diagonally on $\tilde{M} \times \bar{M}$ and $\tilde{N} \times \bar{N}$, respectively. And $\tilde{\phi} \times \bar{\phi}:\left(\tilde{M} \times \bar{M}, \pi_{1} M\right) \rightarrow$ $\left(\tilde{N} \times \bar{N}, \pi_{1} N\right)$ is an $\alpha$-equivariant $C^{0}$-conjugacy of these actions. It induces a homeomorphism between the orbit spaces. Denote this homeomorphism by

$$
\bar{\psi}: \tilde{M} \times_{\pi_{1} M} \bar{M} \rightarrow \tilde{N} \times_{\pi_{1} N} \bar{N} .
$$

Note that projection onto the first factors determine bundles

$$
\tilde{M} \times_{\pi_{1} M} \bar{M} \rightarrow M \text { and } \tilde{N} \times_{\pi_{1} N} \bar{N} \rightarrow N
$$

with fibers $\bar{M}$ and $\bar{N}$; denote these bundles by $\bar{\eta}_{M}$ and $\bar{\eta}_{N}$, respectively. Also $\bar{\psi}$ is a bundle map between them covering $\phi: M \rightarrow N$. Because $\tilde{M}$ and $\tilde{M}(\infty)$ are $\pi_{1}(M)$-invariant subspaces of $\bar{M}$, they determine subbundles of $\bar{\eta}_{M}$; which we denote by $\eta_{M}$ and $\eta_{M}^{\infty}$, respectively. In particular, the total spaces of $\eta_{M}$ and $\eta_{M}^{\infty}$ are $\tilde{M} \times_{\pi_{1} M} \tilde{M}$ and $\tilde{M} \times_{\pi_{1} M} \tilde{M}(\infty)$. There are likewise subbundles $\eta_{N \text {. }}$ $\eta_{N}^{\infty}$ of $\bar{\eta}_{N}$ with fibers $\tilde{N}, \tilde{N}(\infty)$, respectively. The bundle map $\bar{\psi}$ respects these subbundles because of property 1 above. Let $\psi$ and $\psi_{\infty}$ denote the induced bundle maps; i.e.,

$$
\psi=\left.\bar{\psi}\right|_{\tilde{M} \times_{\pi_{1} M} \tilde{M}} \text { and } \psi_{\infty}=\left.\bar{\psi}\right|_{\tilde{M} \times_{\pi_{1} M} \tilde{M}(\infty)} .
$$

We recall that the exponential map for $\tilde{M}$ induces a smooth equivalence between the tangent bundle $T M$ and $\eta_{M}$ via the $\pi_{1} M$ equivariant map

$$
v \mapsto\left(\gamma_{v}(0), \gamma_{v}(1)\right)
$$

from $T \tilde{M} \rightarrow \tilde{M} \times \tilde{M}$. (Remember from section 0 that $\gamma_{v}$ denotes the geodesic in $\tilde{M}$ such that $\dot{\gamma}_{v}(0)=v$ where $v \in T \tilde{M}$. Also, $\exp (v)=\gamma_{v}(1)$.) The smooth bundles $T N$ and $\eta_{N}$ are identified in the same way. Under these identifications $\psi$ represents the tangent microbundle map $d \phi: T M \rightarrow T N$ induced by the homeomorphism $\phi$. Kirby-Siebenmann smoothing theory from [21, Theorem 10.1 , p. 194] yields that $\phi$ is homotopic to a diffeomorphism $f: M \rightarrow N$ provided $d \phi$ is homotopic via topological bundle maps covering $\phi$ to an affine bundle map; i.e., a bundle map which is an affine map on each fiber.

The next result is a first step towards accomplishing this smoothing criterion. We denote the set of all zero vectors in a vector bundle by $\overline{0}$. 
Lemma 1.1. The map $d \phi$ is homotopic through topological bundle maps covering $\phi$ to a map $h$ such that $h(\overline{0})=\overline{0}, h(S M)=S N$ and $h$ is continuously differentiable on $T M-\overline{0}$ with respect to vectors tangent to the fibers of $T M \rightarrow M$. Furthermore, $h$ restricts to a $C^{1}$-diffeomorphism from $T_{x} M-0$ to $T_{\phi(x)} N-0$ for each $x \in M$.

Proof. Let $p: E \rightarrow B$ be a fiber bundle with fiber $F$. We recall that the fiberwise cone on this bundle $\xi$ is a fiber bundle $c \xi$ defined by $q: \mathcal{E} \rightarrow B$ where $\mathcal{E}$ is the quotient space of $E \times[0,+\infty]$ with points $\left(e_{1}, 0\right)$ and $\left(e_{2}, 0\right)$ identified when $p\left(e_{1}\right)=p\left(e_{2}\right)$. The map $q$ is induced by composition of $p$ with projection onto the first factor of $E \times[0,+\infty]$. The fiber of $c \xi$ is the cone on $F$; i. e. $c F$. And $\xi$ is identified with the subbundle of $c \xi$ determined by $E \times+\infty$. There is also a canonical cross section $c: B \rightarrow \mathcal{E}$ where $c(x)$ is the cone point of the fiber $q^{-1}(x)$; i.e., $c(x)$ corresponds to the set $p^{-1}(x) \times 0$. Let $\bar{c}$ denote the image of this cross section.

Since $\bar{M}$ has the cone topology, we can identify $\bar{\eta}_{M}$ with $c \eta_{M}^{\infty}$. This identification is determined as follows. To each pair $v \in S \tilde{M}$ and $r \in[0,+\infty]$, we associate the points in the total spaces of $\bar{\eta}_{M}$ and $c \eta_{M}^{\infty}$ which are to be identified. The pair $\left(\gamma_{v}(0), \gamma_{v}(r)\right) \in \tilde{M} \times \bar{M}$ determines the point in the total space of $\bar{\eta}_{M}$. The triple $\left(\gamma_{v}(0), \gamma_{v}(+\infty), r\right) \in \tilde{M} \times \tilde{M}(\infty) \times[0,+\infty]$ determines the corresponding point in the total space of $c \eta_{M}^{\infty}$.

Under this identification $T M=\eta_{M}$ becomes the complementary subbundle to $\eta_{M}^{\infty}$ in $c \eta_{M}^{\infty}$. Likewise, $\bar{\eta}_{N}$ and $c \eta_{N}^{\infty}$ are identified so that

$$
T N=\eta_{N}=c \eta_{N}^{\infty}-\eta_{N}^{\infty} .
$$

The fiberwise cone of the bundle map $\psi_{\infty}$ is a bundle map $c \psi_{\infty}: c \eta_{M}^{\infty} \rightarrow c \eta_{N}^{\infty}$ induced by the map $\psi_{\infty} \times \operatorname{id}_{[0,+\infty]}$. With the above identifications, it restricts to a bundle map $h: T M \rightarrow T N$ covering $\phi$. This is the map posited to exist in Lemma 1.1.

Let $E_{M}$ and $E_{N}$ denote the total spaces of $c \eta_{M}^{\infty}$ and $c \eta_{N}^{\infty}$, respectively. The natural $C^{1}$-structures on $\tilde{M}(\infty)$ and $\tilde{N}(\infty)$ together with the smooth structures on $\tilde{M}$ and $\tilde{N}$ induce $C^{1}$-structures on $E_{M}-\bar{c}$ and $E_{N}-\bar{c}$, respectively, making $E_{M}-\bar{c} \rightarrow M$ and $E_{N}-\bar{c} \rightarrow N$ into $C^{1}$-fiber bundles. Note also that 
$c \psi_{\infty}(\bar{c})=\bar{c}$. Hence condition 2 of Theorem 0.2 shows that $c \psi_{\infty}$ is a homeomorphism between these $C^{1}$-manifolds which is continuously differentiable with respect to vectors tangent to the fibers of $E_{M}-\bar{c} \rightarrow M$. Furthermore for each $x \in M, c \psi_{\infty}$ maps the fiber of $E_{M}-\bar{c} \rightarrow M$ over $x$ diffeomorphically to the fiber of $E_{N}-\bar{c} \rightarrow N$ over $\phi(x)$. The identifications of $T M$ and $T N$ with subbundles of $c \eta_{M}^{\infty}$ and $c \eta_{N}^{\infty}$ send $\overline{0}$ homeomorphically to $\bar{c}$. And these identifications give $C^{1}$-diffeomorphisms of $T M-\overline{0}$ and $T N-\overline{0}$ with the interiors of $E_{M}-\bar{c}$ and $E_{N}-\bar{c}$, respectively. Consequently, $h(\overline{0})=\overline{0}$ and $h$ is continuously differentiable on $T M-\overline{0}$ with respect to vectors tangent to the fibers of $T M \rightarrow M$. Furthermore, $h$ maps $T_{x} M-0$ diffeomorphically to $T_{\phi^{\prime}(x)} N-0$ for each $x \in M$.

Under the above identifications of $\bar{\eta}_{M}$ with $c \eta_{M}^{\infty}$ and $\bar{\eta}_{N}$ with $c \eta_{N}^{\infty}, \bar{\psi}$ becomes a bundle map $c \eta_{M}^{\infty} \rightarrow c \eta_{N}^{\infty}$ covering $\phi$ and agreeing with $\psi_{\infty}$ on $\eta_{M}^{\infty}$. Let $F_{1}$ and $F_{2}$ be a pair of compact spaces and $\bar{g}: c F_{1} \rightarrow c F_{2}$ be a homeomorphism such that $\bar{g}\left(F_{1}\right)=F_{2}$. Let $g_{\infty}=\left.\bar{g}\right|_{F_{1}}$ and $c g_{\infty}: c F_{1} \rightarrow c F_{2}$ be the cone on $g_{\infty}$. Recall the Alexander isotopy is a canonical (topological) isotopy from $\bar{g}$ to $c g$ which is pointwise fixed on $F_{1}$. Since it is canonical, we can apply it fiberwise to the bundle maps $\psi, c \psi_{\infty}: c \eta_{M}^{\infty} \rightarrow c \eta_{N}^{\infty}$. This yields a homotopy through bundle maps covering the homeomorphism $\phi: M \rightarrow N$ between $\bar{\psi}$ and $c \psi_{\infty}$. Restricting this homotopy to the subbundle $T M$, then yields the homotopy through topological bundle maps covering $\phi$ between $d \phi$ and $h$ which is posited to exist in Lemma 1.1.

We now assume additionally that $\chi(M)=0$. Hence, the tangent bundle $T M \rightarrow M$ has a non-zero cross section $\sigma$; i.e., $\sigma(M) \cap \overline{0}=\emptyset$. Use $\sigma$ to define an affine bundle map $A: T M \rightarrow T N$ covering $\phi$ as follows. For each $x \in M$, the derivative of $h$ at $\sigma(x)$ in the direction of $T_{x} M$ is a linear transformation $L_{x}: T_{x} M \rightarrow T_{\phi(x)} N$. It determines a vector $u_{x} \in T_{\phi(x)} N$ by the formula

$$
u_{x}=h(\sigma(x))-L_{x}(\sigma(x)) .
$$

Let $A_{x}: T_{x} M \rightarrow T_{\phi(x)} N$ be the affine map given by

$$
A_{x}(v)=L_{x}(v)+u_{x}
$$

for all $v \in T_{x} M$. Then, $A$ is the bundle map defined by $\left.A\right|_{T_{x} M}=A_{x}$. 
A parametrized version of a result of Stewart [25] produces a homotopy $h_{t}$ of topological bundle maps covering $\phi$ between $A=h_{0}$ and $h=h_{1}$. It is explicitly given by the following formula in which $x \in M, v \in T_{x} M$ and $t \in[0,1]:$

$$
h_{t}(v)= \begin{cases}A_{x}(v), & \text { if } t=0 \\ \frac{1}{t}[h(t(v-\sigma(x))+\sigma(x))-h(\sigma(x))]+h(\sigma(x)), & \text { if } t \in(0,1] .\end{cases}
$$

Hence, the Kirby-Siebenmann smoothing criterion is satisfied proving Theorem 0.2 .

We now drop the assumption that $\chi(M)=0$ and discuss the modifications in the above argument needed to prove Addendum 0.3. There is still a cross section $\sigma: M \rightarrow T M$ to the bundle projection $p$ which meets $\overline{0}$ in a single point; i.e., $\sigma(M) \cap \overline{0}=\sigma(*)$ for some point $* \in M$. Recall that the homotopy sphere $\Sigma$ in 0.3 can be constructed by gluing $B_{*} M$ to $B_{\phi(*)} N$ via

$$
\left.h\right|_{S_{*} M}: S_{*} M \rightarrow S_{\phi(*)} N
$$

cf. Remark 0.3.1. Hence $\Sigma$ is diffeomorphic to $S^{m}$ (where $m=\operatorname{dim} M$ ) if and only if $\left.h\right|_{S_{*} M}$ extends to a $C^{1}$-diffeomorphism from $B_{*} M$ to $B_{\phi(*)} N$. We now prove 0.3 under the extra assumption that $\Sigma$ is diffeomorphic to $S^{m}$. Then we can modify the map $h$ of Lemma 1.1 by using the previous sentence to obtain a bundle map $\hat{h}: T M \rightarrow T N$ with the following properties:

1. $\hat{h}$ is homotopic to $h$ through topological bundle maps covering $\phi$.

2. There exist open neighborhoods $U \subset V$ of $*$ in $M$ such that $\hat{h}$ and $h$ agree on $p^{-1}(M-V)$.

3. $\hat{h}$ is continuously differentiable on $(T M-\overline{0}) \cup p^{-1}(U)$ with respect to vectors tangent to the fibers of $p: T M \rightarrow M$.

4. For each $x \in M, \hat{h}$ maps $T_{x} M-0$ diffeomorphically onto $T_{\phi(x)} M-0$.

5. For each $x \in U, \hat{h}$ maps $T_{x} M$ diffeomorphically onto $T_{\phi(x)} M$.

Now define an affine bundle map $A: T M \rightarrow T N$ covering $\phi$ as in the proof of Theorem 0.2 but using $\hat{h}$ in place of $h$. Then, replacing $h$ with $\hat{h}$ in formula (1.2) yields a homotopy of topological bundle maps covering $\phi$ between $A$ and 
$\hat{h}$. This means that the conditions of the Kirby-Siebenmann smoothing criterion are again satisfied; thus proving Addendum 0.3 when $\Sigma$ is diffeomorphic to $S^{m}$. The general case is handled similarly by an obstruction theory argument using these techniques in a more detailed manner. The fleshing out of this argument is left to the reader.

\section{ApPlication}

Throughout the remainder of this paper, we specialize to the situation where both $M$ and $N$ are strictly negatively curved. Let $\left(S M, g^{t}\right)$ denote the geodesic flow $g^{t}$ on the total space $S M$ of the unit tangent sphere bundle of $M ;\left(S N, g^{t}\right)$ is likewise the geodesic flow on $S N$. This is the basic example of an Anosov flow [1]. Recall that $\left(S M, g^{t}\right)$ and $\left(S N, g^{t}\right)$ are $C^{1}$-orbit conjugate provided there is a $C^{1}$-diffeomorphism $\Lambda: S M \rightarrow S N$ which maps the orbits of $\left(S M, g^{t}\right)$ to orbits in $\left(S N, g^{t}\right)$ so that the time orientations are preserved. If the orbit preserving map $\Lambda$ is only a homeomorphism, then the two flows are $C^{0}$-orbit conjugate. The flows are $C^{r}$-conjugate $(r=0,1)$ provided

$$
\Lambda\left(g^{t}(v)\right)=g^{t}(\Lambda(v))
$$

for all $v \in S M, t \in \mathbb{R}$ and $\Lambda$ is a $C^{1}$ - diffeomorphism when $r=1$ and a homeomorphism when $r=0$. Clearly, $C^{r}$-conjugacy implies $C^{r}$-orbit conjugacy.

Observe that a $C^{0}$-orbit conjugacy induces an isomorphism $\alpha: \pi_{1}(M) \rightarrow$ $\pi_{1}(N)$. (Recall $\operatorname{dim} M \geq 5$.) Gromov [12] showed conversely that any isomorphism $\alpha: \pi_{1}(M) \rightarrow \pi_{1}(N)$ is induced by a $C^{0}$-orbit conjugacy. We now reformulate Corollary 0.4 in terms of $C^{1}$-orbit conjugacy.

Theorem 2.1. Suppose that $\alpha$ is induced by a $C^{1}$-orbit conjugacy and that both $\tilde{M}(\infty)$ and $\tilde{N}(\infty)$ are naturally $C^{1}$. Then $\alpha$ is also induced by a smooth diffeomorphism $f: M \# \chi(M) \Sigma \rightarrow N$ where $\Sigma$ is the homotopy sphere constructed from $\alpha_{\infty}$ via the procedure given in Remark 0.3.1.

Proof. It is well known that these assumptions imply that $\alpha_{\infty}$ is a $C^{1}$-diffeomorphism and hence Theorem 2.1 follows from Corollary 0.4. But we supply a proof that $\alpha_{\infty}$ is a $C^{1}$-diffeomorphism for the reader's convenience. Let $\Lambda$ be 
the $C^{1}$-orbit conjugacy inducing $\alpha$ and $g: M \rightarrow N$ be a homotopy equivalence also inducing $\alpha$. Consider the diagram

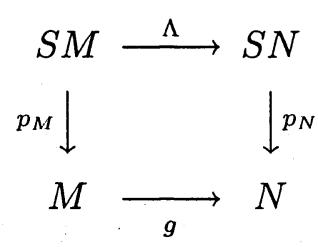

where $p_{M}, p_{N}$ are the bundle projections. This diagram is homotopy commutative since $N$ is a $K\left(\pi_{1} N, 1\right)$. Let $h: S M \times[0,1] \rightarrow N$ be the homotopy between $p_{N} \circ \Lambda$ and $g \circ p_{M}$. Let $\tilde{\Lambda}: S \tilde{M} \rightarrow S \tilde{N}$ be a lift of $\Lambda$. Note that $\tilde{\Lambda}$ is also a $C^{1}$-orbit conjugacy. Let $\tilde{h}: S \tilde{M} \times[0,1] \rightarrow \tilde{N}$ be the lift of $h$ starting at $p_{\tilde{N}} \circ \tilde{\Lambda}$ where $p_{\tilde{N}}: S \tilde{N} \rightarrow \tilde{N}$ is the bundle projection. A simple covering space argument shows that there exists a lift $\tilde{g}: \tilde{M} \rightarrow \tilde{N}$ of $g$ such that $\tilde{h}$ is a homotopy between $p_{\tilde{N}} \circ \tilde{\Lambda}$ and $\tilde{g} \circ p_{\tilde{M}}$; i.e., $\tilde{g}$ makes the following diagram homotopy commutative

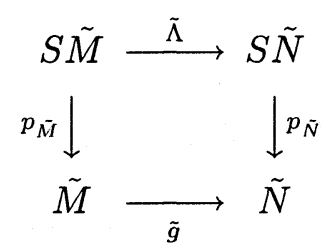

where $p_{\tilde{M}}$ is also the bundle projection. An important consequence of this is the following.

Fact 2.1.1. The set of real numbers $d\left(p_{\tilde{N}}(\tilde{\Lambda}(u)), \tilde{g}\left(p_{\tilde{M}}(u)\right)\right)$ where $u \in S \tilde{M}$ is bounded above.

We see from this that for each $u \in S \tilde{M}$ the curves

$$
p_{\tilde{N}}\left(\tilde{\Lambda}\left(g^{t} u\right)\right) \text { and } \tilde{g}\left(p_{\tilde{M}}\left(g^{t} u\right)\right)
$$

where $t \in[0,+\infty)$ stay a finite distance apart. And consequently conclude that

$$
\alpha_{\infty}(F(u))=F(\tilde{\Lambda}(u))
$$

for each $u \in S \tilde{M}$ where $F: S \tilde{M} \rightarrow \tilde{M}(\infty)$ and $F: S \tilde{N} \rightarrow \tilde{N}(\infty)$ are the 
canonical maps in Definition 0.1; i.e., the following diagram commutes

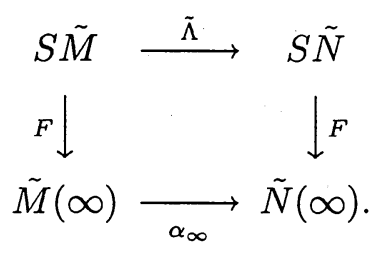

(See the characterization of $\alpha_{\infty}$ in the paragraph preceding Corollary 0.4.) It follows that $\alpha_{\infty}$ is a $C^{1}$-map since both $\tilde{M}(\infty)$ and $\tilde{N}(\infty)$ are naturally $C^{1}$; cf. Definition 0.1. An analogous argument shows that $\left(\alpha^{-1}\right)_{\infty}$ is also $C^{1}$. We conclude that $\alpha_{\infty}$ is a $C^{1}$-diffeomorphism since $\left(\alpha_{\infty}\right)^{-1}=\left(\alpha^{-1}\right)_{\infty}$.

Remark 2.1.2. Theorem 2.1 and Corollary 0.4 are essentially the same result since $C^{1}$-orbit conjugacy is in fact known to be equivalent to $\alpha_{\infty}$ being a $C^{1}$-diffeomorphism when the Anosov splittings of TSM, TSN are $C^{1}$.

Feres [11] proved the following criterion for establishing that $\tilde{M}(\infty)$ is naturally $C^{1}$.

Lemma 2.2. If either the weak stable or the weak unstable foliation for the geodesic flow on $S M$ is $C^{1}$, then $\tilde{M}(\infty)$ is naturally $C^{1}$.

Remark 2.2.1. Hirsch and Pugh [18] showed that the Anosov foliations in $S M$ are $C^{1}$ when $M$ is strictly 1/4-pinched. This result combined with 2.2 shows that $\tilde{M}(\infty)$ is naturally $C^{1}$ when $M$ is strictly $1 / 4$-pinched.

Proof of Theorem 0.5. Hamenstädt [14] showed that any isomorphism $\alpha$ of marked length spectra is induced by a $C^{0}$ - conjugacy $\Lambda$ between the geodesic flows $\left(S M, g^{t}\right)$ and $\left(S N, g^{t}\right)$ inducing $\alpha$. She showed in [15] that $\Lambda$ is a $C^{2}$ diffeomorphism provided the Anosov foliations in $S M$ and $S N$ are $C^{1}$. Combined with Remark 2.2.1, Hamenstädt's results yield a $C^{1}$-orbit conjugacy between $\left(S M, g^{t}\right)$ and $\left(S N, g^{t}\right)$ inducing $\alpha$ since $M$ and $N$ are assumed to be strictly 1/4-pinched. Theorem 0.5 now follows from Theorem 2.1 since $\tilde{M}(\infty)$ and $\tilde{N}(\infty)$ are naturally $C^{1}$ because of Remark 2.2.1.

Remark 2.3. The above argument shows that the conclusion of 0.5 still holds when the assumption that $M$ and $N$ are strictly 1/4-pinched is replaced by the weaker assumption that the Anosov foliations in $S M$ and $S N$ are $C^{1}$. 


\section{REFERENCES}

1. Anosov, D. V., Geodesic flows on closed Riemann manifolds with negative curvature, Proc. Steklov Inst. Math. 90 (1967).

2. Ballman, W., Gromov, M. and Schroeder, V., Manifolds of Nonpositive Curvature, Progr. Mathematics, vol. 61, Birkhäuser, 1985.

3. Besson, Courtis and Gallot, Les varétês hyperboliques sont les minima de l'entropie topologique, preprint.

4. Corlette, K., Archimedean superrigidity and hyperbolic geometry, Ann. of Math, 135 (1992), 165-182.

5. Eberlein, P., Rigidity of lattices of nonpositive curvature, Ergodic Theory and Dynamical Systems 3 (1983), 47-85.

6. Eberlein, P., Hamenstädt, U. and Schroeder, V., Manifolds of nonpositive curvature, Proc. Sympos. Pure Math., vol. 54, part 3, Amer. Math. Soc., Providence, R.I., 1993, pp. 179-227.

7. Eberlein, P. and O'Neill, B., Visibility manifolds, Pacific J. Math. 46 (1973), 45-109.

8. Farrell, F.T. and Jones, L.E., Negatively curved manifolds with exotic smooth structures, J. Amer. Math. Soc. 2 (1989), 899-908.

9. Farrell, F.T. and Jones, L.E., Topological rigidity for compact nonpositively curved manifolds, Proc. Sympos. Pure Math., vol. 54, part 3, Amer. Math. Soc., Providence, R.I., 1993, pp. 229-274.

10. Farrell, F.T. and Jones, L.E., Complex hyperbolic manifolds and exotic smooth structures, Invent. Math. 117 (1994), 57-74.

11. Feres, R., Geodesic flows on manifolds of negative curvature with smooth horospheric foliations, Ergodic Th. Dynamical Systems 11 (1991), 653-686.

12. Gromov, M., Hyperbolic groups, Essays in Group Theory (S.M. Gersten, ed.), Math. Sci. Res. Inst. Publ., vol. 8, Springer-Verlag, 1987, pp. 75-263.

13. Gromov, M., Foliated plateau problem, Geom. Funct. Anal. to appear.

14. Hamenstädt, U., Time preserving conjugacies of geodesic flows Ergodic Theory Dynamical Systems 12 (1992), 67-74.

15. Hamenstädt, U., Regularity of time-preserving conjugacies for contact Anosov flows with $C^{1}$-Anosov splitting, Ergodic Theory Dynamical Systems 13 (1993), 65-72.

16. Hamenstädt, U., Harmonic measures for compact negatively curved manifolds and rigidity, preprint.

17. Hernández, L., Kähler manifolds and 1/4-pinching, Duke J. Math. 62 (1991), 601-611.

18. Hirsch, M. and Pugh, C., Smoothness of horocycle foliations, J. Differential Geom. 10 (1975), 225-238.

19. Kanai, M., Geodesic flows on negatively curved manifolds with smooth stable and unstable foliations, Ergodic Theory Dynamical Systems 8 (1988), 215-239.

20. Kervaire, M. and Milnor, J., Groups of homotopy spheres: I, Ann. of Math.77 (1963), 504-537.

21. Kirby, R.C. and Siebenmann, L.C., Foundational essays on topological manifolds, 
smoothings, and triangulations, Ann. of Math. Stud., no. 88, Princeton Univ. Press, Princeton, N.J., 1977.

22. Mostow, G.D., On the conjugacy of subgroups of semi-simple groups, Proc. of Symposia in Pure Math. vol. 9 (1966), pp. 413-419.

23. Mostow, G.D., Strong rigidity of locally symmetric spaces, Ann. of Math. Stud., no. 78, Princeton Univ. Press, Princeton, N.J., 1973.

24. Ontaneda, P., Hyperbolic manifolds with negatively curved exotic triangulations in dimension six, J. Differential Geom. 117 (1994), 57-74.

25. Stewart, T.E., On groups of diffeomorphisms, Proc. Amer. Math. Soc. 11 (1960), 559-563.

26. Yau, S.-T. and Zheng, F., Negatively 1/4-pinched riemannian metric on a compact Kähler manifold, Invent. Math. 103 (1991), 527-535.

SUNY, BinghamTon, U. S. A.

SUNY, STONY BROOK, U. S. A.

RECEIVED APRIL 15, 1994. 